\title{
Efficient Genome Editing by a Miniature CRISPR-AsCas12f1 Nuclease in Bacillus anthracis
}

\author{
Yanchun Wang*, Shuli Sang, Xin Zhang, Haoxia Tao, Qing Guan and Chunjie Liu* \\ State Key Laboratory of Pathogens and Biosecurity, Institute of Biotechnology, Academy of Military Medical Sciences, Beijng, \\ China
}

A miniature CRISPR-Cas12f has been demonstrated to serve as an effective genome editing tool in gram negative bacteria as well as human cells. Here, we developed an alternative method to edit the genome of Bacillus anthracis based on the AsCas12f1 nuclease from Acidibacillus sulfuroxidans. When the htrA gene on the chromosome and the lef gene on the plasmid pXO1 were selected as targets, the CRISPR-AsCas12f1 system showed very high efficiency (100\%). At the same time, a high efficiency was observed for large-fragment deletion. Our results also indicated that the length of the homologous arms of the donor DNA had a close relationship with the editing efficiency. Furthermore, a two-plasmid CRISPR-

Edited by: Tao Chen,

Tianjin University, China

Reviewed by:

Quanjiang Ji,

ShanghaiTech University, China

Chaoyou Xue,

Tianjin Institute of Industrial

Biotechnology (CAS), China

${ }^{*}$ Correspondence:

Yanchun Wang

springwyc@163.com

Chunie Liu

liucj@nic.bmi.ac.cn

Specialty section: This article was submitted to

Synthetic Biology,

a section of the journal Frontiers in Bioengineering and

Biotechnology

Received: 01 December 2021 Accepted: 27 December 2021 Published: 14 January 2022

Citation:

Wang $Y$, Sang S, Zhang X, Tao H, Guan Q and Liu C (2022) Efficient Genome Editing by a Miniature CRISPR-AsCas $12 f 1$ Nuclease in

Bacillus anthracis.

Front. Bioeng. Biotechnol. 9:825493.

doi: $10.3389 /$ fbioe. 2021.825493 AsCas12f1 system was also constructed and combined with the endonuclease I-Scel for potential multi-gene modification. This represents a novel tool for mutant strain construction and gene function analyses in B. anthracis and other Bacillus cereus group bacteria.

Keywords: CRISPR-Cas12f, Bacillus anthracis, genome editing, endonuclease I-Scel, plasmid curing

\section{INTRODUCTION}

Clustered regularly interspaced short palindromic repeats (CRISPRs) are part of the bacterial immune system that defends against invading viruses (Westra et al., 2014). They are made up of repeating sequences of genetic code that are interrupted by pieces of genetic code from previous invaders, which allows a cell to detect and destroy returning invaders. Based on this characteristic, CRISPR-associated (Cas) nucleases have been extensively used in genome editing in many species and an increasing number of newly discovered Cas nucleases have been explored as novel genome editing tools (Jiang and Marraffini, 2015; Manghwar et al., 2019). To date, the miniature CRISPR effectors, such as Cas $\Phi$, Cas13, and Cas14, have been developed into functional genome editors (Aquino-Jarquin, 2019; Savage, 2019; Pausch et al., 2020; Awan et al., 2021). Of these Cas nucleases, Cas12f has been shown to be superior as an editing system due to its small molecular weight and ease of cellular delivery (Awan et al., 2021).

Cas12f (also known as Cas14) is a family of relatively compact RNA-guided nucleases that were originally found in an uncultivated archaea species. It belongs to the class 2 type V-F CRISPR-associated effector nuclease family. Most Cas12f proteins are approximately 400-700 amino acids in length, include a single RuvC nuclease domain, and are known as miniature Cas proteins (Takeda et al., 2021; Xiao et al., 2021). Cas $12 \mathrm{f}$ was discovered by mining a database of microbial genomes and metagenomes, and Cas $12 \mathrm{f}$ was shown to bind and cleave a targeted ssDNA sequence using a specific gRNA sequence. However in this work, they did not determine if Cas12f was able to target dsDNA both in vivo and in vitro (Harrington et al., 2018). Next, Karvelis et al. confirmed that Cas $12 \mathrm{f}$ nucleases recognize and cleave dsDNA in a TTTR (where R is A or G) PAM-dependent manner and have the potential to be harnessed as programmable nucleases for genome editing (Karvelis et al., 2020). Currently, Cas $12 \mathrm{f}$ has been demonstrated to serve as an effective genome editing tool in bacteria as well as human cells. 
Okano et al. showed that the Un1Cas12f1 (529 aa) from an uncultured archaeon (Un1) could modify the Escherichia coli genome with high efficiency (50-100\%) (Okano et al., 2021). Kim et al. showed that an optimized CRISPR/Un1Cas12f1 system enabled efficient and specific genome editing in human cells, with efficiency and specificity similar to that of SpCas9 and AsCas12a (Kim et al., 2021). Wu et al. also showed that AsCas12f1 from Acidibacillus sulfuroxidans could serve as an effective genome editing tool in both bacteria and human cells. Moreover, this AsCas12f1 system could be delivered by various delivery methods, including plasmid, ribonucleoprotein and adenoassociated virus (Wu et al., 2021). At the same time, Xu et al. developed a miniature CRISPR system with Cas12f mutants, named CasMINI, which enabled robust gene editing and base editing in mammalian cells (Xu et al., 2021). Bigelyte et al. showed that SpCas12f1 functioned in both plant and human cells to produce targeted modifications (Bigelyte et al., 2021). All of these works provide sufficient evidence that Cas12f can be developed a useful tool for broad genome engineering applications.

In a previous study, we developed a genome-editing protocol for $B$. cereus group strains based on the CRISPRCas9 system (Wang et al., 2019). Both large fragment deletion and precise point mutations could be achieved efficiently. Unfortunately, the large size of SpCas9 (1368 amino acids) is a nonnegligible problem, and this increases the size of any genome editing plasmid using SpCas9. Like many bacteria, the transformation efficiency of $B$. anthracis decreases with the increasing of plasmid size, and this also is a significant problem in many poorly transformable strains (Ohse et al., 1995; Turgeon et al., 2006). Alternative, miniature Cas nucleases would be a better choice in $B$. anthracis and other similar strains.

In the present study, the feasibility of CRISPR-Cas $12 \mathrm{f}$ in $B$. anthracis was determined. When the $h \operatorname{tr} A$ gene on the $B$. anthracis chromosome and the lef gene on the plasmid pXO1 were selected as targets, $100 \%$ modification rates were achieved in these experiments. Then the effect of homologous arm length on editing efficiency also was explored by comparative analysis of the results using plasmids with different lengths of homologous arms. At the same time a two-plasmid CRISPR-AsCas12f1 system was also constructed and combined with endonuclease I-SceI for potential multigene modification.

Taken together, an efficient genome editing protocol for $B$. anthracis was developed based on the CRISPR-AsCas12f1 system. This protocol will be a useful tool for mutant strain construction and gene function analyses in B. anthracis.

\section{MATERIALS AND METHODS}

\section{Bacterial Strains, Plasmids, and Growth Conditions}

All bacterial strains and plasmids used in this study are listed in Table 1. Escherichia coli Top 10 cells were used as a cloning host, and $E$. coli SCS110 was used to prepare unmethylated plasmids. $E$. coli was grown aerobically in $\mathrm{LB}$ medium at $37^{\circ} \mathrm{C}$, while $B$. anthracis was grown in BHIG medium (brain heart infusion broth with the addition of $0.5 \%$ glycerol). Kanamycin $(50 \mu \mathrm{g} / \mathrm{ml}$ for E. coli, $30 \mu \mathrm{g} / \mathrm{ml}$ for B. anthracis) or erythromycin $(400 \mu \mathrm{g} / \mathrm{ml}$ for $E$. coli, $5 \mu \mathrm{g} / \mathrm{ml}$ for $B$. anthracis) was added to the media at appropriate final concentrations as needed.

\section{Editing Plasmid Construction}

All constructed plasmids used in this work are shown in Table 1, and PCR primers and the N20 sequences for each PAM sequence (TTTG) are listed in Table 2. All the sequence information of synthetic DNA fragments is supplied in supplementary information (Supplementary Table S1). B. anthracis-codonoptimized AsCas12f1 from A. sulfuroxidans with the B. subtilis mannose manP promoter (PmanP) and the sgRNA_V1 fragment designed according to a reference were ordered from General Biosystems (China). To introduce the CRISPR-AsCas12f1 system into a plasmid, the Cas9 cassette of plasmid pJOE8999 was replaced by this synthetic AsCas12f1 cassette, and the EcoRI$X b a I$ fragment of the resulting plasmid was replaced with the synthetic sgRNA sgRNA_V1 fragment. This plasmid was designated pJOE-Cas12f1.

To explore the feasibility of CRISPR-Cas $12 \mathrm{f}$ system in $B$. anthracis, the htrA gene on the $B$. anthracis chromosome and the lef gene on the plasmid pXO1 were selected as targets. The 800-bp upstream and downstream regions of these target genes were amplified using $B$. anthracis A16R genomic DNA as a template and inserting it into the corresponding SalI and $\mathrm{XbaI}$ sites of pJOE-Cas12f1. For the htrA gene, primers UhtrAF/ UhtrAR and DhtrAF/DhtrAR were used, respectively. Then the small double stranded target spacer, annealed with the two complementary oligonucleotides (sg-htrAF1/sg-htrAR1or sghtrAF2/sg-htrAR2), was inserted in the location between the two BsaI sites of the plasmid to obtain gene specific genome editing plasmids. In this work, two sgRNAs with different target spacers on each gene were designed and tested respectively in subsequent experiments. In the same manner, plasmids for lef gene deletion were constructed accordingly.

To test the effect of the length of homologous arms on the editing efficiency, a series of $h t r A$ specific genome editing plasmids with varying lengths $(50,100,200$, or $400 \mathrm{bp})$ of upstream and downstream regions were constructed using a similar method and also were used to delete the $h \operatorname{tr} A$ gene.

To explore the feasibility of large chromosomal fragment deletions using the CRISPR-Cas12f system in B. anthracis, the prophage lambdaBa03 $(\sim 16.8 \mathrm{~kb})$ was selected as the target. The plasmids for lambdaBa03 deletion were constructed accordingly in the similar way. The upstream and downstream regions of lambdaBa03 were amplified using plasmid pJOE-lam03 constructed previously as template (Wang et al., 2019). In this part, two different lengths (400 bp and $800 \mathrm{pb}$ ) upstream and downstream regions were designed and tested the editing efficiency.

\section{Genome Editing With a Single Plasmid}

The transformation and selection of competent cells was performed as described previously (Wang et al., 2019). pJOEcas $12 \mathrm{f} 1$ series plasmids were introduced by electroporation of $B$. 
TABLE 1 | Plasmids and strains used in this study.

\begin{tabular}{|c|c|c|}
\hline Plasmids and strains & Relevant characteristics & Source \\
\hline \multicolumn{3}{|l|}{ Plasmids } \\
\hline pJOE8999 & Rep pE194 (Ts), Kanr, Pman-cas9, PvanP*-sgRNA9, shuttle vector & Altenbuchner, (2016) \\
\hline pJOE-Cas12f1 & Rep pE194 (Ts), Kanr, Pman-AsCas12f1, PvanP*-sgRNA12, shuttle vector & This study \\
\hline pJOE-Cas12f1-htrA-1 & pJOE-Cas $12 \mathrm{f1}$ with sgRNA1-htrA and homologous arms of $h$ trA from $B$. anthracis A16R & This study \\
\hline pJOE-Cas12f1-htrA-1 & pJOE-Cas12f1 with sgRNA2-htrA and homologous arms of $h$ trA from $B$. anthracis A16R & This study \\
\hline pJOE-Cas12f1-lef-1 & pJOE-Cas12f1 with sgRNA1-lef and homologous arms of lef from B. anthracis A16R & This study \\
\hline pJOE-Cas12f1-lef-2 & pJOE-Cas12f1 with sgRNA2-lef and homologous arms of lef from B. anthracis A16R & This study \\
\hline pJOE-Lam03 & pJOE8999 with Cas9 and homologous arms of lam03 from B. anthracis A16R & Wang et al. (2019) \\
\hline pJOE-Cas12f1-lam03-400 & pJOE-Cas12f1 with sgRNA and $400 \mathrm{bp}$ homologous arms of prophage lambdaBa03 from $B$. anthracis A16R & This study \\
\hline pJOE-Cas12f1-lam03-800 & pJOE-Cas $12 \mathrm{f} 1$ with sgRNA and $800 \mathrm{bp}$ homologous arms of prophage lambdaBa03 from $B$. anthracis A16R & This study \\
\hline $\mathrm{pHY304}$ & shuttle vector, resource of ermAM, Erm ${ }^{r}$ & Jeng et al. (2003) \\
\hline pSS4332 & shuttle vector, expressing endonuclease I-Scel & Cybulski et al. (2009) \\
\hline pJOE-mScarlet & Deriving from pJOE8999, from 1 to 2844 base pairs, carrying mScarlet coded sequence without promoter & This study \\
\hline pCas12f1-Scel-E & Rep pE194 (Ts), Kanr, Pman-AsCas12f1, PxylA-I-Scel, Erm², shuttle vector & This study \\
\hline pSS-FD & modified skeleton of pSS4332, PvanP*-sgRNA12, two I-Scel sites, shuttle vector & This study \\
\hline pSS-FD-htrA & pSS-FD with sgRNA-lef and homologous arms of htrA from $B$. anthracis A16R & This study \\
\hline \multicolumn{3}{|l|}{ B. anthracis strains } \\
\hline B. anthracis A16R & pXO1+pXO2-, China vaccine strain, host for genome editing & This laboratory \\
\hline B.anthracis A16R $\Delta h t r A$ & B.anthracis A16R with htrA gene deletion & This study \\
\hline B.anthracis A16R $\Delta l e f$ & B.anthracis A16R with lef gene deletion & This study \\
\hline B.anthracis A16R_lam03 & B.anthracis A16R excision prophage lambdaBa03 & This study \\
\hline \multicolumn{3}{|l|}{ E. coli strains } \\
\hline $\mathrm{DH} 5 a$ & Cloning strain & CWBIO, China \\
\hline SCS110 & dam-/dcm- strain used to produce unmethylated plasmid & Transgen, China \\
\hline
\end{tabular}

anthracis $\mathrm{A} 16 \mathrm{R}$, and transformants were selected at $30^{\circ} \mathrm{C}$ on BHIG medium containing kanamycin. Single colonies were transferred to liquid media (with $25 \mu \mathrm{g} / \mathrm{ml}$ kanamycin) and incubated with shaking for $3 \mathrm{~h}$ at $37^{\circ} \mathrm{C}$. Mannose (final concentration, $0.4 \%$ ) was added to induce the expression of the Cas12f1 protein. After $3 \mathrm{~h}$ of cultivation, serial dilutions of this culture were plated on LB agar with $25 \mu \mathrm{g} / \mathrm{ml}$ kanamycin and $0.4 \%$ mannose and then incubated at $37^{\circ} \mathrm{C}$ overnight. Transformants were identified by colony PCR and DNA sequencing. For PCR tests, the B. anthracis A16R strain was used as a negative control.

\section{Inducible Promoter Screening}

All alternative promoter fragments and mScarlet coding sequence were designed according to reference or sequence information from the NCBI database and ordered from GeneralBio Co. (China). To construct the promoter screening plasmid, the plasmid pJOE8999 was digested with BsrGI and EcoRI to release the backbone of the plasmid (from 1 to $2844 \mathrm{bp}$ ). Then the synthetic coding region of the $\mathrm{mScarlet}$ coding sequence with applicable restriction sites was ligated with this fragment to construct plasmid pJOE-mScarlet (Bindels et al., 2017). Next, alternative promoters, the bacitracin-inducible promoter (Toymentseva et al., 2012), cumate-inducible promoter (Seo and Schmidt-Dannert, 2019), or xylose-inducible promoter from Bacillus megaterium and Bacillus subtilis, were inserted upstream of the mScarlet coding sequence and the resulting plasmids were introduced into the $B$. anthracis A16R strain and selected at $30^{\circ} \mathrm{C}$ on BHIG medium containing kanamycin.

For comparison of the mScarlet expression levels of $B$. anthracis A16R harboring different recombinant plasmid, $5 \mathrm{ml}$ of LB cultures were grown to an OD600 $\mathrm{nm}=0.6-1.0$, then the suitable inducers were added to induce target protein expression. Cultures without inducers were used as a negative control. After 10 hours of induction, the OD600 $\mathrm{nm}$ and fluorescence intensity of cultures (excitation at $569 \mathrm{~nm}$ and emission at $594 \mathrm{~nm}$ ) were measured with a SpectraMax®i3x.

\section{Two-Plasmid System Editing Plasmid Construction}

To cure CRISPR plasmids as soon as possible, a two-plasmid system based on CRISPR-Cas12f was also constructed. At first, the I-SceI cassette (containing the xylose induced promoter and the coding region of I-SceI) was inserted at a location between the $X b a \mathrm{I}$ and EcoRI sites in plasmid pJOE-Cas12f1 by Gibson assembly. The resulting plasmid was digested with XhoI and PstI to remove the kanamycin resistance cassette, then the erythromycin resistance cassette amplified from pHY304 was cloned into this plasmid to replace the kanamycin resistance cassette. The resulting plasmid was designated pCas12f1-SceI-E.

At the same time, the I-SceI coding region of the modified plasmid pSS4332 (a point mutation was introduced to destroy the $B s a \mathrm{I}$ site in $\operatorname{repB}$ of this plasmid) was replaced by a synthetic DNA fragment, including the sgRNA_V1 cassette, restriction sites for homologous arm insertion, and two I-SceI sites on both sides, to construct plasmid pSS-FD. Then the homologous arms and the target spacer of the $h t r A$ gene were inserted into the plasmid pSS-FD successively using the previously described method. The resulting plasmid was designated pSS-FD-htrA and used for genome editing.

\section{Genome Editing With Two Plasmids}

For the two-plasmid system, B. anthracis A16R competent cells harboring pCas12f1-SceI plasmid were prepared using previously 
TABLE 2 | Primers used in this study.

\begin{tabular}{|c|c|c|}
\hline Name & Sequence $\left(5^{\prime} \rightarrow 3^{\prime}\right)$ & Purpose \\
\hline UtrAF & ACGCGTCGACGACTATAGTITGGC & PCR of homology arms for htrA deletion \\
\hline UtrAR & TाTGGTCTCGTAAACTCGGAATAAAAGAAAGTCTC & - \\
\hline DtrAF & TाTGTCTCGTTACTTCCССTCTCTG & - \\
\hline DtrAR & CTAGCTAGCTCGAAGCAGAAGACG & - \\
\hline sg-htrAF1 & GAACGTTAAATAACGCACCACCAC & space sequence 1 of $h$ trA \\
\hline sg-htrAR1 & GGCCGTGGTGGTGCGTTATITAAC & - \\
\hline sg-htrAF2 & GAACCATCTACCTTCTTGCCATCA & space sequence 2 of $h$ trA \\
\hline sg-htrAR2 & GGCCTGATGGCAAGAAGGTAGATG & - \\
\hline htrAiF & GAAACCATATACGATGTACGTTCTGG & - \\
\hline htrAiR & AAGATGAAAGAAGATTACGTGAAATTG & PCR of htrA deletion identification \\
\hline UefF & ACGCGTCGACAGATGTGGTGGGCAAG & PCR of homology arms for lef deletion \\
\hline UlefR & CGGGATCCGTAATGTATTAAAAATITCAAATG & - \\
\hline DlefF & CGGGATCCATTAATCTCTCCTIITATAAG & - \\
\hline DlefR & CTAGCTAGCAAATCAATGCGTAAATTGACC & - \\
\hline sg-lefF1 & GAACGCACTACTITCGCATCAATC & space sequence 1 of lef \\
\hline sg-lefR1 & GGCCGATTGATGCGAAAGTAGTGC & - \\
\hline sg-lefF2 & GAACGCTCAATAGGAATCTGCAGC & space sequence 2 of lef \\
\hline sg-lefR2 & GGCCGCTGCAGATTCCTATTGAGC & - \\
\hline lefiF & GAAATGGTCAGCACCGCCAGAAG & PCR of lef deletion identification \\
\hline lefiR & TGTGTCTAATGTAGCAGATACATCTAG & - \\
\hline lam03-800F & ACGCGTCGACGGAGAATTCTाTGAAG & PCR of 800 bp homology arms for lambdaBa03 excision \\
\hline lam03-800R & GCTCTAGAAGTTGGTGCTCCAACATTC & - \\
\hline lam03-400F & ACGCGTCGACGGTAGCCCCTTCCATGA & PCR of $400 \mathrm{bp}$ homology arms for lambdaBa03 excision \\
\hline lam03-400R & GCTCTAGAAACTGAGCGTATCGGTGA & - \\
\hline sg-lam03F & GAACCTGACGAGCCTAACCCACGA & space sequence of lambdaBa03 \\
\hline sg-lam03R & GGCCTCGTGGGTTAGGCTCGTCAG & - \\
\hline lam03iF & CCTGGGATTGATGATACGATGG & PCR of lambdaBa03 excision identification \\
\hline lam03iR & GAAGCAATCGCTCCAGAAATCG & - \\
\hline htrA-400F & ACGCGTCGACTACTCCTAATTGTGCCC & PCR of $400 \mathrm{bp}$ homology arms for htrA \\
\hline htrA-400R & TGCTCTAGAGAACTTCTCGTTTATITAATG & - \\
\hline htrA-200F & ACGCGTCGACTTGCTITGAAACCATATAC & PCR of $200 \mathrm{bp}$ homology arms for htrA \\
\hline htrA-200R & TGCTCTAGACGGAACGTATTGTGTGCTTC & - \\
\hline htrA-100F & ACGCGTCGACAGTTGGGTITCAATTGTC & PCR of $100 \mathrm{bp}$ homology arms for htrA \\
\hline htrA-100R & TGCTCTAGATAAGCGTATIIITAATTGG & - \\
\hline htrA-50F & ACGCGTCGACCCGATAAAGAAAGTCTC & PCR of 50 bp homology arms for htrA \\
\hline htrA-50R & TGCTCTAGAAAAACGTGACTATACTGAA & - \\
\hline rScelF & TाTCПIITGCGTGTGATGCGCTAATAACATATAAACAGCCAGTTG & PCR of PxylA-I-Scel cassette \\
\hline rScelR & ATATITAGATGAAGATTATTCTTAATCAAAAAACCCCTCAAGACCCG & - \\
\hline ErmF & AACTGCAGACAAATCACTTATCACAAATC & PCR ofermAM cassette from pHY304 \\
\hline ErmR & CCGCTCGAGCCTCTITAGCTCCTTGGAAGC & - \\
\hline
\end{tabular}

described methods, and then the pSS-FD-htrA plasmid was introduced by electroporation. Genome editing was triggered by inducing AsCas12f1 expression. Briefly, a strain harboring pCas12f1-SceI and pSS-FD-thrA was transferred to liquid media (with $25 \mu \mathrm{g} / \mathrm{ml}$ kanamycin and $5 \mu \mathrm{g} / \mathrm{ml}$ erythromycin), and incubated with shaking for $3 \mathrm{~h}$ at $37^{\circ} \mathrm{C}$. Mannose (final concentration, $0.4 \%$ ) was added to induce the expression of the Cas12f1 protein. After cultivation for $3 \mathrm{~h}$, serial dilutions of this culture were plated on LB agar with erythromycin and $0.4 \%$ mannose and cells were incubated at $37^{\circ} \mathrm{C}$ overnight. Transformants were identified by colony PCR and the $B$. anthracis A16R strain was used as a negative control.

\section{Plasmid Curing}

To cure the pSS-FD-htrA plasmid, an edited colony harboring both pCas12F and pSS-FD-htrA was transferred to liquid BHIG media (with $5 \mu \mathrm{g} / \mathrm{ml}$ erythromycin), and incubated with shaking for $3 \mathrm{~h}$ at $37^{\circ} \mathrm{C}$. Xylose (final concentration, $0.4 \%$, w/v) was added to induce the expression of the endonuclease I-SceI and the culture without xylose was used as negative control. After cultivation for $3 \mathrm{~h}$, the culture was diluted and spread onto BHIG plates with $5 \mu \mathrm{g} / \mathrm{ml}$ erythromycin and $0.4 \%$ xylose, and these plates were incubated at $37^{\circ} \mathrm{C}$ for $16-20 \mathrm{~h}$. The curing of plasmid of pSS-FD-htrA was confirmed by determining the colony sensitivity to kanamycin $(25 \mu \mathrm{g} / \mathrm{ml})$.

\section{RESULTS}

\section{Establishment and Improvement of the CRISPR-AsCas12f1 Genome Editing System}

For demonstration of the feasibility of the CRISPR-Cas12f system in B. anthracis, the plasmid pJOE-AsCas12f1 was constructed and employed for genome editing (Figure 1A). First, as described in 

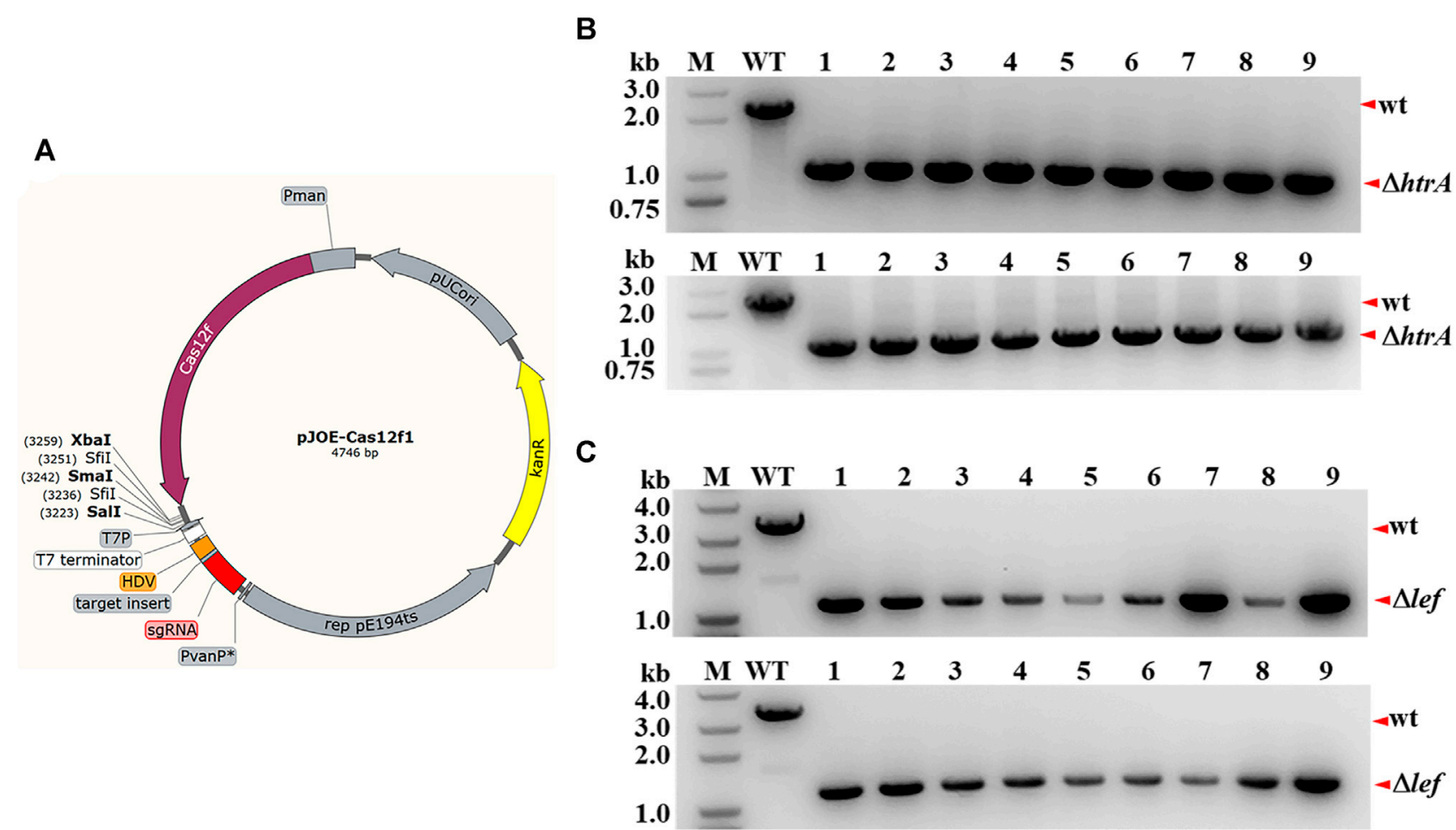

FIGURE 1 | Gene deletion via the CRISPR-CRISPR-AsCas12f1 system in B. anthracis. (A) Physical map of plasmid pJOE-cas12f1. Pman, PmanP promoter; PvanP $^{\star}$, semisynthetic promoter PvanP*. The cloning sites and insertion site of the spacer sequence (Bsal restriction sites) are also labeled in the map. (B) PCR verification of $h$ trA deletion in $B$. anthracis A16R. The $h$ trA gene locus of the randomly selected colonies carrying the editing plasmids was amplified, and the PCR products were verified through agarose gel analysis. M, DNA marker; WT, control with B.anthracis A16R total DNA as the template; "wt" and " $\Delta$ htrA", wild-type band and the $h$ trA-deleted band, respectively. The correct fragment in the mutant strain was approximately 1.0 kb (lanes 1-9) while in A16R this was 2.3 kb (lane WT). (C) PCR verification of lef deletion in B. anthracis A16R. "wt" and " $\Delta /$ lef", wild-type band and the lef-deleted band, respectively. The correct fragment in the mutant strain was approximately $1.2 \mathrm{~kb}$ (lanes 1-9) while in A16R this was $3.6 \mathrm{~kb}$ (lane WT).

Materials and methods section, the plasmid including donor DNA, gene-specific sgRNA, and AsCas12f1 cassettes was electroporated into B. anthracis A16R. Then the genome editing events were performed as described previously (Wang et al., 2019). The htrA gene on the $B$. anthracis chromosome and the lef gene on the plasmid pXO1 were selected as targets, and alternative mutants were detected by colony PCR. For the htrA gene, compared to the $2.3 \mathrm{~kb}$ amplicon from the parental strain A16R, the $1.0 \mathrm{~kb}$ PCR products from all randomly selected colonies (9/9 and 9/9 for each target spacer) showed that $h \operatorname{tr} A$ (1242 bp) had been deleted successfully (Figure 1B). A similar result was also seen for lef deletion (Figure 1C). These results confirmed the predominant feasibility of AsCas12f1 for B. anthracis genome editing.

To study the relationship between the editing efficiency and the length of homologous arms, four other editing plasmids with different lengths of homologous arms (50, 100, 200, and $400 \mathrm{bp})$ were constructed. The results of $h t r A$ gene deletion indicated that the length of the homologous arms was closely related to the editing efficiency (Figure 2A). PCR tests indicated that when the lengths of the homologous arms were $50 \mathrm{bp}$ or $100 \mathrm{bp}$, none of the 30 randomly selection colonies showed $h \operatorname{tr} A$ gene deletion bands and only wild type amplification bands (Figures 2B,C). When the length of the homologous arms was $200 \mathrm{bp}$, only 2 of the 15 randomly selection colonies exhibited the $h \operatorname{tr} A$ gene deletion band while most colonies (13/15) were heterozygous (two bands amplification, Figure 2D). When the length of the homologous arms was 400 or $800 \mathrm{bp}, 100 \%$ of randomly selection colonies (15/ 15 and 15/15, Figures 2E,F) showed that $h t r A$ had been completely deleted (pure deletion type).

To test the availability of the CRISPR-cas12f system for longer DNA fragment deletion, the prophage lambdaBa03 was excised by similar method. 9 randomly selection colonies were screened via PCR. The region amplified by the lamiF/lamiR primers in the control strain A16R was $>19 \mathrm{~kb}$ in length, which exceeded the maximum amplification size under our PCR conditions. However, When the length of the homologous arms was $800 \mathrm{bp}$, all randomly selected colonies (9/9) showed the expected $2.2 \mathrm{~kb}$ amplicon, indicating that the prophage lambdaBa03 had been excised successfully (Figure 3A). At the same time, despite the substantially reduced size of the homologous arms, the $100 \%$ editing efficiency (9/9) was also seen when the $400 \mathrm{bp}$ homologous arms were employed (Figure 3B).

\section{Two-plasmid-based CRISPR-Cas12f System and Plasmid Curing}

To strictly induce the expression of the endonuclease I-SceI, four different promoters were selected to drive the expression of the 

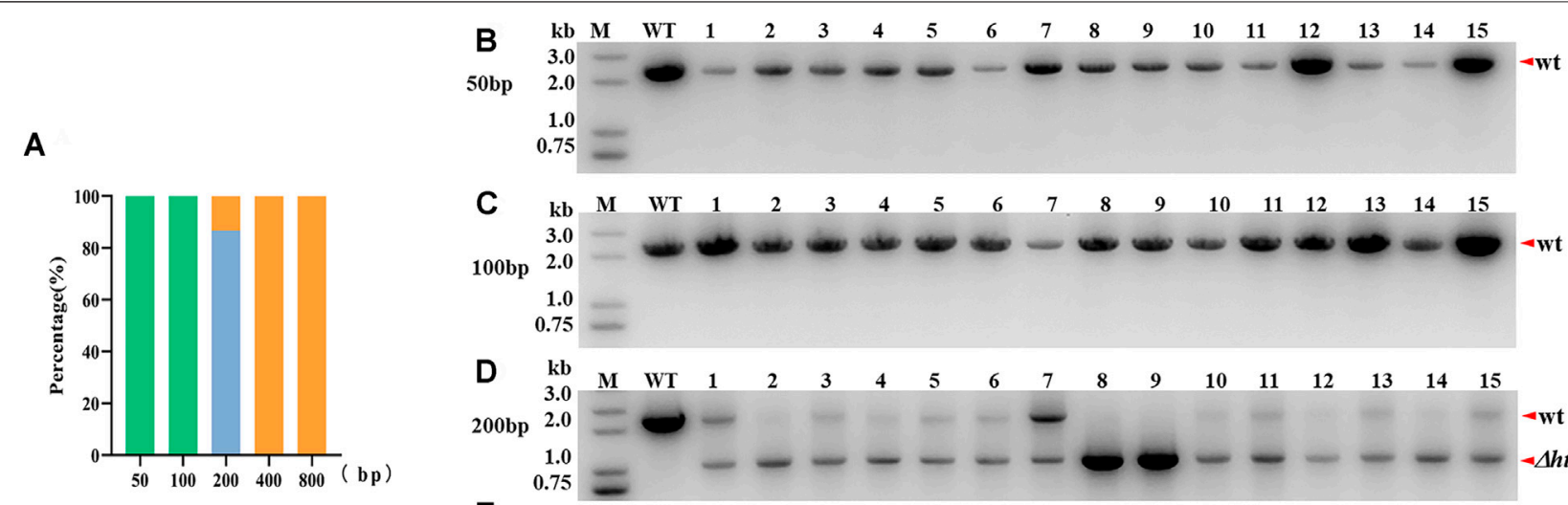

Wild-genotype $\square$ Hetero-type $\square$ Deletion-type

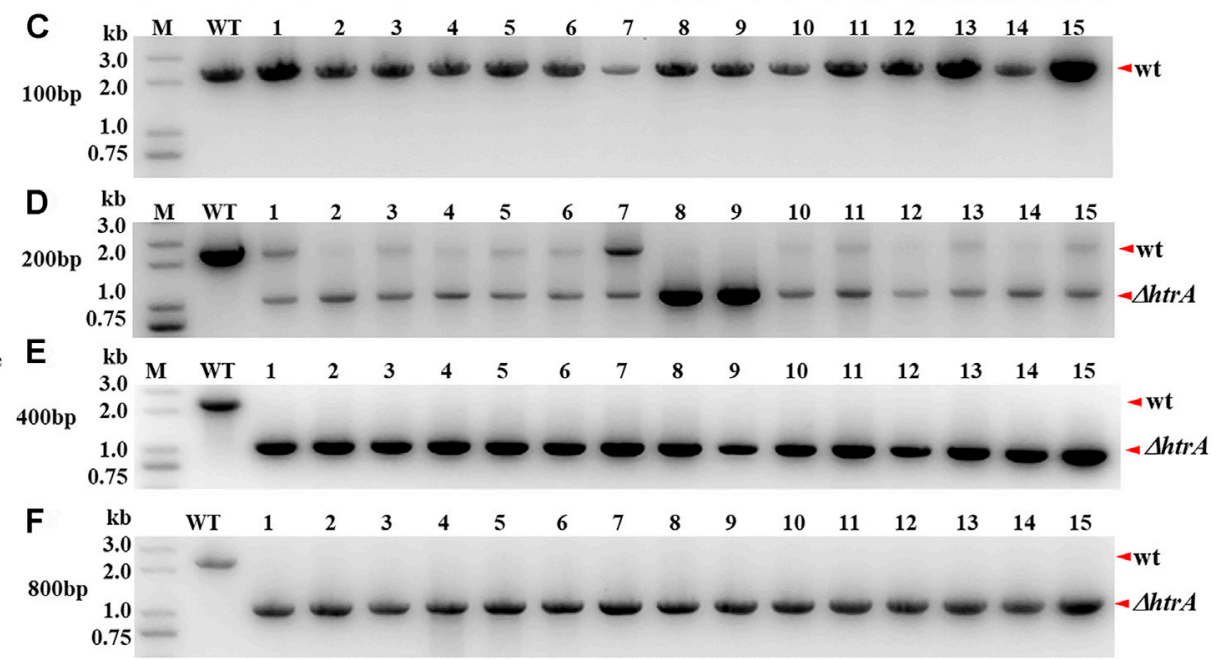

FIGURE 2 | Effect of homologous arm length on gene deletion efficiency. (A) Comparison analysis of the genome editing efficiencies of plasmids with different length homologous arms. Hetero-type colonies showed bands for both wild type and $h$ trA gene deletion. (B-F) PCR validation of $h$ trA gene deletion in $B$. anthracis A16R using editing plasmids with different length homologous arms (50, 100, 200, 400, and 800 bp). M, DNA marker; WT, B. anthracis A16R total DNA as a template; wt, wildtype band; and $\Delta$ trA, htrA-deleted band. For each plasmid group, 15 colonies were selected to validate by PCR and agarose gel analysis.

report protein mScarlet. The results demonstrated that the PxylA promoter from B. subtilis could be strictly induced and the expression level of the report gene was relatively high (Figure 4A). Based on this result, a plasmid co-expressing AsCas12f1 and the endonuclease I-SceI under the control of the PxylA promoter was constructed as show in Figure 4B. Combined with another plasmid pSS-FD carrying the sgRNA cassette, this twoplasmid-based CRISPR-Cas12f system for B. anthracis was successfully established.

To demonstrate the feasibility of this two-plasmid system, the $h t r A$ gene was selected as a target once again and the editing efficiency was tested. As shown in Figure 4C, all nine randomly selected colonies (9/9) showed that htrA (1242 bp) had been deleted in B. anthracis A16R. At the same time, the result of plasmid curing tests indicated that a portion of the recombinant colonies (12/50, 6/50 and 17/50, three independent experiments) had lost kanamycin resistance and the plasmid pSS-FD-htrA had been partially eliminated after endonuclease I-SceI expression was induced by xylose. While no kanamycin sensitive colony was gained in the non-inducible conditions after passage once.

\section{DISCUSSION}

In this work, the CRISPR-Cas12f system was successfully utilized for genome editing in B. anthracis. The efficiency was similar to the CRISPR-SpCas9 system based on our previously reported results (Wang et al., 2019). Either the htrA gene on the $B$. anthracis chromosome or the lef gene on the plasmid pXO1

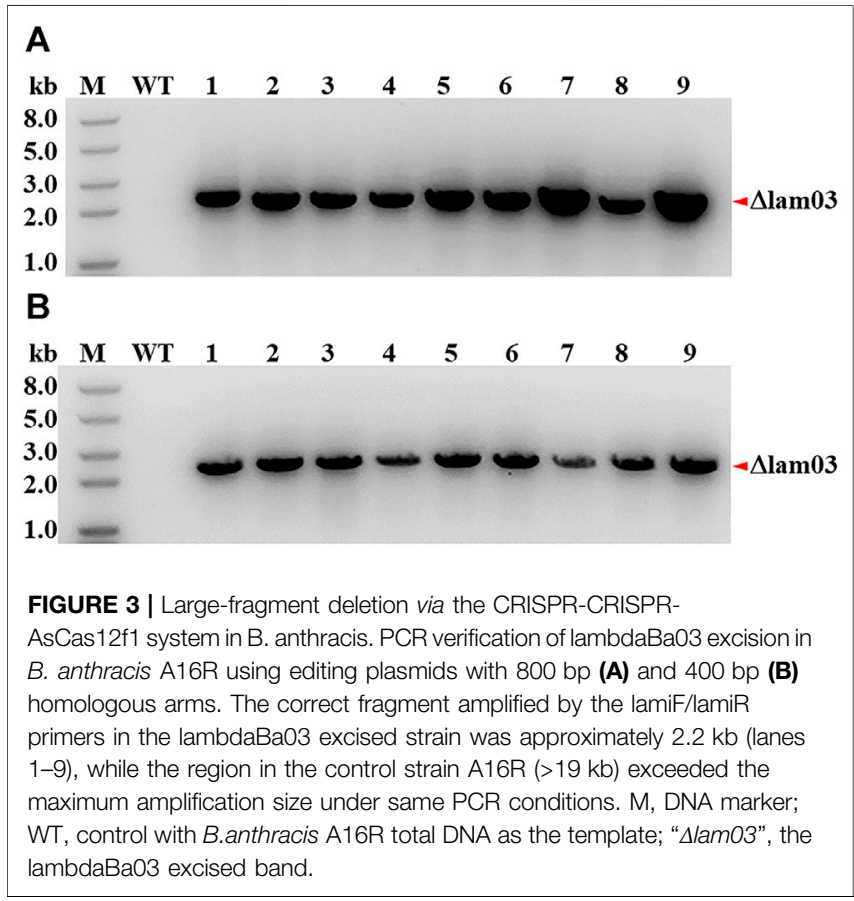

was deleted, and deletion mutants achieved rates of $100 \%$ after one round of induction and selection in these experiments.

At the same time, a high efficiency was seen for large-fragment deletion when prophage lambdaBa03 was selected as target. These 


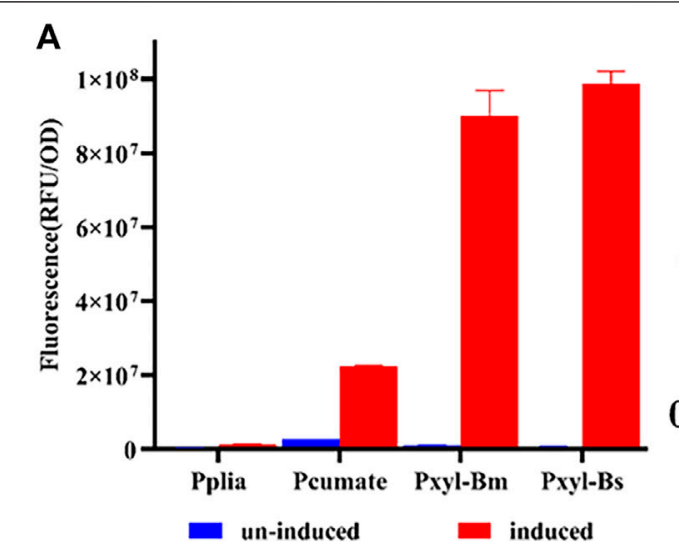

B

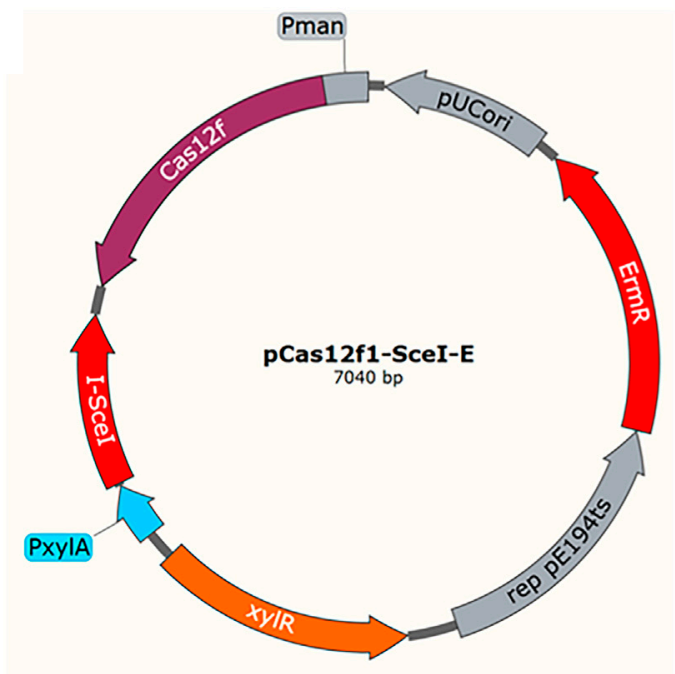

C
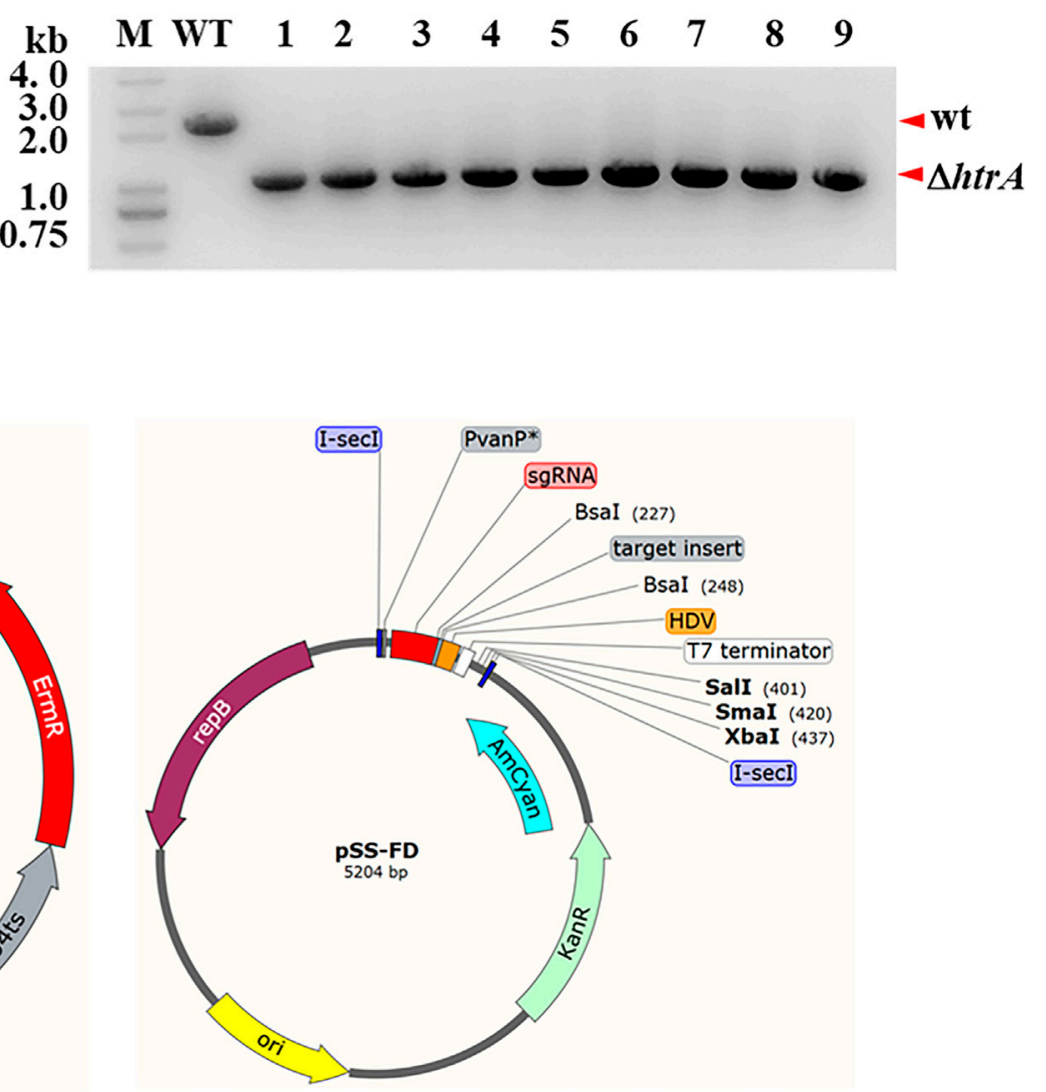

FIGURE 4 | Gene deletion via a two-plasmid CRISPR-CRISPR-AsCas12f1 system in B. anthracis. (A) Induced promoter screening using mScarlet as a reporter molecule. (B) Physical map of the two-plasmid system vectors. pCas12f1-Scel-E, the plasmid co-expressed AsCas12f1 and I-Scel endonuclease I-Scel (under the control of the PxylA promoter); pSS-FD, for sgRNA expression and homologous arm supply. (C) PCR verification of $h$ trA deletion via the two-plasmid system. M, DNA marker; WT, B. anthracis A16R total DNA as a template; wt, wild-type band; and $\Delta h t r A$, htrA-deleted band. The correct fragment in the mutant strain was approximately $1.0 \mathrm{~kb}$ (lanes 1-9) while in A16R this was $2.3 \mathrm{~kb}$ (lane WT).

results indicated that the CRISPR-12f system was a highefficiency genetic operation tool in B. anthracis. Moreover, the protocol developed in this work may be generally applicable to other bacillus group strains.

Compared to the CRISPR-Cas9 system, the CRISPR-Cas12f system had a clear comparative advantage. The AsCas12f1 used in our work consisted of 422 amino acid residues, as this was one of the high-activity miniature CRISPR-Cas effectors (Kim et al., 2021; Wu et al., 2021). This characterization work demonstrated that the size of the genome editing plasmid was smaller and higher transformation efficiency was obtained in B. anthracis relevant experiments using this effector. At the same time, based on published results, the most efficient PAMs recognized by AsCas12f1 were $5^{\prime}$-TTTR (where R represents A or G) (Karvelis et al., 2020). For B. anthrcis, a low-GC-content Gram Positive Bacteria, the distribution of PAMs sequence was thus more general and the spacer screening became easier for many genes. This means that our CRISPR-Cas $12 \mathrm{f}$ system would be more useful in B. anthracis and other low-GC-content bacteria compared to a more traditional SpCas9-based system.

When a one-plasmid-based CRISPR-Cas system was used for two or more gene edits in same parent strain, the rate of editing plasmid curing was shown to be the major constraint and more attention must be paid to overcome this (Wu et al., 2019). According to published studies and our results, some recombinant plasmids, despite including a temperature sensitive origin of replication, are very difficult to cure at the non-permissive temperature in B. anthracis and some other bacillus species (Hartz et al., 2021). When the plasmids derived from pJOE8999 were used to edit the $B$. anthracis genome, 8-10 passages in antibiotic free medium at the non-permissive temperature were frequently needed to derive transformants without editing plasmids. The period of these experiments were thus lengthened (unpublished data). To solve this issue, separate Cas9 and sgRNA on different plasmids using a two-plasmid system has been a popular 
method (Wasels et al., 2017; Ao et al., 2018). Here, we also constructed a two-plasmid CRISPR-Cas12f system combined with endonuclease I-SceI, a tool enzyme can digest plasmids with specific I-SceI sites (TAGGGATAACAGGGTAAT) in vivo (Wang et al., 2018). Two I-SceI sites were introduced into the plasmid with target gene-specific sgRNA and homologous arms. After inducing the expression of the endonuclease I-SceI on the other plasmid, the plasmid with the sgRNA was digested during growth and passage. The final plasmid curing rate was improved substantially using this method. The interval time was thus shortened for the next target gene editing. Although the curing rate of target plasmids in our work was not as high as some other reports (Jiang et al., 2015), in subsequent research, we will investigate the cause of this and test this method rigorously by selecting more genes of interest as disruption targets and confirm that this is a universal method for gene inactivation in $B$. anthracis. Additionally, other plasmid curing methods will also be studied to optimize and improve this current protocol.

\section{REFERENCES}

Altenbuchner, J. (2016). Editing of the Bacillus Subtilis Genome by the CRISPRCas9 System. Appl. Environ. Microbiol. 82 (17), 5421-5427. doi:10.1128/ AEM.01453-16

Ao, X., Yao, Y., Li, T., Yang, T.-T., Dong, X., Zheng, Z.-T., et al. (2018). A Multiplex Genome Editing Method for Escherichia coli Based on CRISPR-Cas12a. Front. Microbiol. 9, 2307. doi:10.3389/fmicb.2018.02307

Aquino-Jarquin, G. (2019). CRISPR-Cas14 Is Now Part of the Artillery for Gene Editing and Molecular Diagnostic. Nanomedicine: Nanotechnology, Biol. Med. 18, 428-431. doi:10.1016/j.nano.2019.03.006

Awan, M. J. A., Amin, I., and Mansoor, S. (2021). Mini CRISPR-Cas12f1: a New Genome Editing Tool. Trends Plant Sci. doi:10.1016/ j.tplants.2021.11.002

Bigelyte, G., Young, J. K., Karvelis, T., Budre, K., Zedaveinyte, R., Djukanovic, V., et al. (2021). Miniature Type V-F CRISPR-Cas Nucleases Enable Targeted DNA Modification in Cells. Nat. Commun. 12 (1), 6191. doi:10.1038/s41467-02126469-4

Bindels, D. S., Haarbosch, L., van Weeren, L., Postma, M., Wiese, K. E., Mastop, M., et al. (2017). mScarlet: a Bright Monomeric Red Fluorescent Protein for Cellular Imaging. Nat. Methods 14 (1), 53-56. doi:10.1038/nmeth.4074

Cybulski, R. J., Jr., Sanz, P., Alem, F., Stibitz, S., Bull, R. L., and O’Brien, A. D. (2009). Four Superoxide Dismutases Contribute to Bacillus Anthracis Virulence and Provide Spores with Redundant protection from Oxidative Stress. Infect. Immun. 77 (1), 274-285. doi:10.1128/IAI.00515-08

Harrington, L. B., Burstein, D., Chen, J. S., Paez-Espino, D., Ma, E., Witte, I. P., et al. (2018). Programmed DNA Destruction by Miniature CRISPR-Cas14 Enzymes. Science 362 (6416), 839-842. doi:10.1126/science.aav4294

Hartz, P., Gehl, M., König, L., Bernhardt, R., and Hannemann, F. (2021). Development and Application of a Highly Efficient CRISPR-Cas9 System for Genome Engineering in Bacillus Megaterium. J. Biotechnol. 329, 170-179. doi:10.1016/j.jbiotec.2021.02.006

Jeng, A., Sakota, V., Li, Z., Datta, V., Beall, B., and Nizet, V. (2003). Molecular Genetic Analysis of a Group A Streptococcus Operon Encoding Serum Opacity Factor and a Novel Fibronectin-Binding Protein, SfbX. J. Bacteriol. 185 (4), 1208-1217. doi:10.1128/JB.185.4.1208-1217.2003

Jiang, W., and Marraffini, L. A. (2015). CRISPR-cas: New Tools for Genetic Manipulations from Bacterial Immunity Systems. Annu. Rev. Microbiol. 69, 209-228. doi:10.1146/annurev-micro-091014-104441

Jiang, Y., Chen, B., Duan, C., Sun, B., Yang, J., and Yang, S. (2015). Multigene Editing in the Escherichia coli Genome via the CRISPR-Cas9 System. Appl. Environ. Microbiol. 81 (7), 2506-2514. doi:10.1128/AEM.04023-14

\section{DATA AVAILABILITY STATEMENT}

The original contributions presented in the study are included in the article/Supplementary Material, further inquiries can be directed to the corresponding authors.

\section{AUTHOR CONTRIBUTIONS}

YW, and CL designed the research. YW, HT, XZ, SS, and QG performed all experiments. YW and CL analyzed data. YW wrote the paper. All authors reviewed the final manuscript.

\section{SUPPLEMENTARY MATERIAL}

The Supplementary Material for this article can be found online at: https://www.frontiersin.org/articles/10.3389/fbioe.2021.825493/ full\#supplementary-material

Karvelis, T., Bigelyte, G., Young, J. K., Hou, Z., Zedaveinyte, R., Budre, K., et al. (2020). PAM Recognition by Miniature CRISPR-Cas12f Nucleases Triggers Programmable Double-Stranded DNA Target Cleavage. Nucleic Acids Res. 48 (9), 5016-5023. doi:10.1093/nar/gkaa208

Kim, D. Y., Lee, J. M., Moon, S. B., Chin, H. J., Park, S., Lim, Y., et al. (2021). Efficient CRISPR Editing with a Hypercompact Cas12f1 and Engineered Guide RNAs Delivered by Adeno-Associated Virus. Nat. Biotechnol. doi:10.1038/ s41587-021-01009-Z

Manghwar, H., Lindsey, K., Zhang, X., and Jin, S. (2019). CRISPR/Cas System: Recent Advances and Future Prospects for Genome Editing. Trends Plant Sci. 24 (12), 1102-1125. doi:10.1016/j.tplants.2019.09.006

Ohse, M., Takahashi, K., Kadowaki, Y., and Kusaoke, H. (1995). Effects of Plasmid DNA Sizes and Several Other Factors on Transformation ofBacillus subtilisISW1214 with Plasmid DNA by Electroporation. Biosci. Biotechnol. Biochem. 59 (8), 1433-1437. doi:10.1271/bbb.59.1433

Okano, K., Sato, Y., Hizume, T., and Honda, K. (2021). Genome Editing by Miniature CRISPR/Cas12f1 Enzyme in Escherichia coli. J. Biosci. Bioeng. 132 (2), 120-124. doi:10.1016/j.jbiosc.2021.04.009

Pausch, P., Al-Shayeb, B., Bisom-Rapp, E., Tsuchida, C. A., Li, Z., Cress, B. F., et al. (2020). CRISPR-Cas $\Phi$ from Huge Phages Is a Hypercompact Genome Editor. Science 369 (6501), 333-337. doi:10.1126/science.abb1400

Savage, D. F. (2019). Cas14: Big Advances from Small CRISPR Proteins. Biochemistry 58 (8), 1024-1025. doi:10.1021/acs.biochem.9b00035

Seo, S.-O., and Schmidt-Dannert, C. (2019). Development of a Synthetic CumateInducible Gene Expression System for Bacillus. Appl. Microbiol. Biotechnol. 103 (1), 303-313. doi:10.1007/s00253-018-9485-4

Takeda, S. N., Nakagawa, R., Okazaki, S., Hirano, H., Kobayashi, K., Kusakizako, T., et al. (2021). Structure of the Miniature Type V-F CRISPR-Cas Effector Enzyme. Mol. Cel 81 (3), 558-570. e553. doi:10.1016/j.molcel.2020.11.035

Toymentseva, A. A., Schrecke, K., Sharipova, M. R., and Mascher, T. (2012). The LIKE System, a Novel Protein Expression Toolbox for Bacillus Subtilis Based on the liaI Promoter. Microb. Cel Fact 11, 143. doi:10.1186/14752859-11-143

Turgeon, N., Laflamme, C., Ho, J., and Duchaine, C. (2006). Elaboration of an Electroporation Protocol for Bacillus Cereus ATCC 14579. J. Microbiol. Methods 67 (3), 543-548. doi:10.1016/j.mimet.2006.05.005

Wang, T., Wang, D., Lyu, Y., Feng, E., Zhu, L., Liu, C., et al. (2018). Construction of a High-Efficiency Cloning System Using the Golden Gate Method and I-SceI Endonuclease for Targeted Gene Replacement in Bacillus Anthracis. J. Biotechnol. 271, 8-16. doi:10.1016/j.jbiotec.2018.02.006

Wang, Y., Wang, D., Wang, X., Tao, H., Feng, E., Zhu, L., et al. (2019). Highly Efficient Genome Engineering in Bacillus Anthracis and Bacillus Cereus Using 
the CRISPR/Cas9 System. Front. Microbiol. 10, 1932. doi:10.3389/ fmicb.2019.01932

Wasels, F., Jean-Marie, J., Collas, F., López-Contreras, A. M., and Lopes Ferreira, N. (2017). A Two-Plasmid Inducible CRISPR/Cas9 Genome Editing Tool for Clostridium Acetobutylicum. J. Microbiol. Methods 140, 5-11. doi:10.1016/ j.mimet.2017.06.010

Westra, E. R., Buckling, A., and Fineran, P. C. (2014). CRISPR-cas Systems: beyond Adaptive Immunity. Nat. Rev. Microbiol. 12 (5), 317-326. doi:10.1038/ nrmicro3241

Wu, Z., Wang, Y., Zhang, Y., Chen, W., Wang, Y., and Ji, Q. (2019). Strategies for Developing CRISPR-Based Gene Editing Methods in Bacteria. Small Methods 4, 1900560. doi:10.1002/smtd.201900560

Wu, Z., Zhang, Y., Yu, H., Pan, D., Wang, Y., Wang, Y., et al. (2021). Programmed Genome Editing by a Miniature CRISPR-Cas12f Nuclease. Nat. Chem. Biol. 17 (11), 1132-1138. doi:10.1038/s41589-021-00868-6

Xiao, R., Li, Z., Wang, S., Han, R., and Chang, L. (2021). Structural Basis for Substrate Recognition and Cleavage by the Dimerization-dependent CRISPR-Cas $12 \mathrm{f}$ Nuclease. Nucleic Acids Res. 49 (7), 4120-4128. doi:10.1093/nar/gkab179

Xu, X., Chemparathy, A., Zeng, L., Kempton, H. R., Shang, S., Nakamura, M., et al. (2021). Engineered Miniature CRISPR-Cas System for Mammalian Genome
Regulation and Editing. Mol. Cel 81 (20), 4333-4345. e4334. doi:10.1016/ j.molcel.2021.08.008

Conflict of Interest: The authors declare that the research was conducted in the absence of any commercial or financial relationships that could be construed as a potential conflict of interest.

Publisher's Note: All claims expressed in this article are solely those of the authors and do not necessarily represent those of their affiliated organizations, or those of the publisher, the editors and the reviewers. Any product that may be evaluated in this article, or claim that may be made by its manufacturer, is not guaranteed or endorsed by the publisher.

Copyright (c) 2022 Wang, Sang, Zhang, Tao, Guan and Liu. This is an open-access article distributed under the terms of the Creative Commons Attribution License (CC $B Y)$. The use, distribution or reproduction in other forums is permitted, provided the original author(s) and the copyright owner(s) are credited and that the original publication in this journal is cited, in accordance with accepted academic practice. No use, distribution or reproduction is permitted which does not comply with these terms. 